\title{
REVIEW
}

\section{Association of response endpoints with survival outcomes in multiple myeloma}

\author{
$\mathrm{S}_{\text {Lonial }}{ }^{1}$ and KC Anderson ${ }^{2}$
}

\begin{abstract}
Since the introduction of the proteasome inhibitor bortezomib and the immunomodulatory drugs (IMiDs) thalidomide and lenalidomide, more patients with multiple myeloma are achieving deep, durable responses and disease control, and are living longer. These improvements have afforded more robust analyses of the relationship between response and survival. Generally, these studies have demonstrated that improvements in the quality of response across all stages of treatment are associated with better disease control and longer survival. Thus, achievement of maximal response should be strongly considered, particularly in the frontline setting, but must also be balanced with tolerability, quality of life and patient preferences. In select patients, achievement of a lesser response may be adequate to prolong survival, and attempts to treat these patients to a deeper response may place them at unnecessary risk without significant benefit. Maintenance therapy has been shown to improve the quality of response and disease control and, in some studies, survival. Studies support maintenance therapy for high-risk patients as a standard of care, and there are emerging data supporting maintenance therapy in standard-risk patients to improve progressionfree and possibly overall survival. Multidrug regimens combining a proteasome inhibitor and an IMiD have shown exceptional response outcomes with acceptable increases in toxicity in both the frontline and salvage settings, and are becoming a standard treatment approach. Moving forward, the use of immunophenotypic and molecular response criteria will be essential in better understanding the impact of highly active and continuous treatment regimens across myeloma patient populations. Future translational studies will help to develop antimyeloma agents to their fullest potential. The introduction of novel targeted therapies, including the IMiD pomalidomide and the proteasome inhibitors carfilzomib and ixazomib (MLN9708), will provide greater options to individualize treatment and help patients achieve a clinically meaningful response.
\end{abstract}

Leukemia (2014) 28, 258-268; doi:10.1038/leu.2013.220

Keywords: myeloma; depth of response; durability of response; survival; minimal residual disease; multidrug treatment

\section{INTRODUCTION}

Over the past decade, the introduction of targeted therapy with the proteasome inhibitor (PI) bortezomib (approved by the US Food and Drug Association in 2003) and immunomodulatory drugs (IMiDs) lenalidomide and thalidomide (both approved in 2006) have vastly improved the outcomes for patients with multiple myeloma (MM). ${ }^{1-3}$ When used alone or as part of combination regimens, these therapies have been shown to provide deep and durable, quality responses, which have translated into advances in disease control and survival. During the early years of their use from 2003 to 2006, the 5-year survival estimate in MM increased to $40.3 \%$ compared with $32.8 \%$ between 1998 and 2002. ${ }^{2}$ Unfortunately, most patients still face the challenges of disease progression and eventually succumb to the disease.

The treatment paradigm for $\mathrm{MM}$ is evolving, but the general goals continue to include rapid disease control to reverse complications, extending disease control and survival, and maintaining quality of life. ${ }^{4-6}$ In recent years, a growing body of evidence indicates a relationship between the quality of response to treatment (depth and durability) and clinical outcomes. Before the introduction of targeted therapies, most patients were unable to achieve quality responses without undergoing high-dose therapy with autologous stem cell transplant (HD-ASCT). Given that HD-ASCT was initially limited to patients under 65 years of age and MM has a median onset of 70 years, ${ }^{5}$ many patients were ineligible for transplantation and instead received a less-intensive treatment. As a consequence, achievement of a deep and durable response was rare and thus, it was challenging to relate response and survival. ${ }^{7-10}$ Furthermore, these studies generally excluded high-risk patients for whom the benefit of achieving and maintaining a complete response $(C R)$ is more evident. ${ }^{11}$

The rapid adoption of targeted therapies has afforded a greater focus on depth and duration of response and their impact on outcomes. $^{12-17}$ Both transplant-eligible and -ineligible patients now achieve quality responses, including CRs, across all phases of treatment (induction, consolidation, maintenance and salvage). ${ }^{18}$ The development of triplet combinations that combine a PI with an IMiD and a corticosteroid or chemotherapy has provided unprecedented levels of response in the frontline and relapsed settings in high-risk patients. ${ }^{19-25}$ Generally, studies with targeted therapies support an aggressive treatment paradigm to maximize the quality of response and minimize the burden of the malignant clone, particularly in the early treatment phase. ${ }^{26-32}$ Even at the time of presentation multiple clones can be present. Theoretically, an aggressive upfront treatment strategy may improve the depth and durability of response and inhibit clonal evolution, but clinical studies are needed to support this premise. ${ }^{33-35}$ 
However, there are important considerations. ${ }^{6,36,37}$ Quality of response alone is not a validated surrogate marker of overall survival (OS). ${ }^{38}$ There are currently no definitive data to validate the association between quality of response and survival outcomes. Moreover, the association of depth of response with outcomes is not universal across studies. Biologically, MM is a spectrum of diseases and its course and response to therapy can be highly variable. Some patients achieve very good initial responses that are short-lived, whereas others achieve only minimal responses (MRs) that are durable and clinically relevant. ${ }^{39-41}$ Furthermore, aggressive treatment can be associated with greater toxicity than conventional approaches, and thus, the risk-to-benefit must be compared with alternative approaches that favor 'disease control' with less-intensive sequential regimens. ${ }^{6,37}$ Both strategies have merit but in the context of specific patient populations and disease characteristics. Risk-adapted strategies favor aggressive treatment in high-risk patients and more conventional approaches in standard-risk patients. ${ }^{5}$ However, risk stratification is not sufficiently accurate to predict the quality of a treatment response for individual patients. Undertreatment could result in failure to achieve a potential cure in standard-risk patients or rapid loss of disease control in high-risk patients. For these reasons, it may be preferable to maximize the response of all patients at diagnosis and use personalized treatment in the consolidation and maintenance phases.

In view of the evolving treatment strategies in $M M$, it is important to consider the impact depth of response has on clinical outcomes collectively and within the various treatment phases and disease settings. Although current data do not address all gaps in our knowledge, they do help to better inform treatment decisions. Here, we provide an overview of studies that have explored the impact of quality of response on outcomes by transplant status and stage of treatment, and consider our knowledge gaps. We review recent studies of triplet combinations and highlight the need for more sensitive response criteria that will better inform short- and long-term treatment decisions.

\section{DEPTH OF RESPONSE AND CLINICAL OUTCOMES}

A number of caveats to the data reviewed here should be acknowledged upfront. It is important to keep in mind that before 1998 multiple response criteria were in use with variable definitions for CR or no definition at all. ${ }^{9,10,42,43}$ In 1998, the European Group for Bone Marrow and Blood Transplantation developed the first uniform response criteria for $M M$ based on: monoclonal paraprotein serum and urine levels with electrophoresis and immunofixation; the proportion of plasma cells in the bone marrow aspirates/biopsies with histologic and cytologic studies; bone lesions and soft tissue plasmacytomas, with radiographic imaging and clinical evaluations. ${ }^{44}$ Response categories included $C R$, partial response $(P R), M R$, stable disease and progressive disease. Then, in 2006, the International Myeloma Working Group developed response criteria similar to that of European Group for Bone Marrow and Blood Transplantation but with some differences, including the addition of stringent $C R$ and very good partial response (VGPR) response categories. ${ }^{45}$ Studies also sometimes use near $\mathrm{CR}(\mathrm{nCR})$ as an additional response category. $^{46}$

We also acknowledge that many of the studies discussed here are retrospective, observational or exploratory analyses with limited number of patients. Patient populations and treatments were more homogeneous and well controlled in some studies than in others. Also, survival data may be confounded by the impact of subsequent or salvage therapies at the time of progression, ${ }^{1,3,47-49}$ which may vary based on the depth and duration of the initial response and access to treatments. Analysis of outcomes by the level of response may introduce selection bias, with standard-risk patients being more likely to achieve a quality response than high-risk patients. It is not surprising that studies have reported no association between depth of response and outcomes, but generally these studies were conducted before the advent of the targeted therapy era., ${ }^{9,42,50-53}$

In the targeted therapy era, more patients across the myeloma spectrum are achieving quality responses with improved survival outcomes. In a meta-analysis of phase 3 bortezomib trials that included 2086 transplant-eligible patients, ${ }^{54}$ the use of bortezomib during induction significantly improved overall response rate (ORR), ₹VGPR and CR compared with non-bortezomib regimens, which corresponded to improvements in progression-free survival (PFS) and OS. Despite the lack of definitive data, the body of evidence linking quality of response to outcomes is growing and has become more compelling.

\section{Newly diagnosed MM}

Several studies have assessed the impact of response levels on outcomes in patients with newly diagnosed MM (NDMM) receiving HD-ASCT (Table 1). In general, studies show that a quality response at each treatment stage-induction, HD-ASCT, and consolidation-is associated with improved outcomes. $^{19,26,55,56}$ Lahuerta et al. ${ }^{26}$ evaluated response in 632 patients with NDMM treated with the GEM2000 protocol, which used a chemotherapy induction regimen followed by HD-ASCT. Post transplantation, there was a significant association between response level and outcomes with a 5-year OS rate of $74 \%$ in patients achieving a CR compared with $63 \%$ for nCR $(P=0.01), 50 \%$ for PR $(P<0.0001)$ and $57 \%$ for stable disease $(P=0.01)$. Post induction, there were trends of improved survival by level of response, but the association was less robust. There was no difference in OS for achievement of CR post induction versus post transplantation. Outcomes were worse for patients with persistent $\mathrm{nCR}$ (before and after transplantation) compared with patients whose response improved to nCR post transplantation with a 5 -year OS rate of $45 \%$ versus $72 \%$ $(P=0.001)$. In a separate phase 3 IFM 2005-01 study, which randomized 482 patients to induction with bortezomib and dexamethasone or vincristine, doxorubicin and dexamethasone (VAD), PFS was significantly longer for patients achieving a VGPR post induction compared with post transplantation (median 41.2 versus 31.1 months; $P=0.01){ }^{27}$ In multivariate analysis, a response $\leqslant$ VGPR overall and post induction were both retained as negative prognostic factors. Taken together, data from these and other studies $^{26,27,55,56}$ suggest that it is important to attain a quality response early with induction therapy and drive the response further in the subsequent treatment stages.

Studies have also demonstrated the prognostic implications of response durability. ${ }^{51,57}$ Barlogie et al. ${ }^{57}$ conducted a retrospective analysis of OS by response at a 36-month landmark in patients enrolled in a total therapy (TT)2 study (double HD-ASCT supported by induction, consolidation and maintenance with thalidomide; $n=668)$. Response categories included $C R$ sustained for $\geqslant 36$ months $(n=256)$, CR lost before 36 months $(n=39)$ and non-CR $(n=211)$. Median OS was significantly longer in the sustained $C R$ group (not reached) versus the non-CR group (5.6 years, $P<0.0001)$ and the lost-CR group (1.6 years, $P<0.0001)$, as well as for the comparison of non-CR to lost-CR $(P<0.0001)$. These survival trends were also observed in patients enrolled in a TT1 study as well as a cohort receiving other transplant protocols. Similarly, a pooled analysis of GEM2000 and GEM2005 studies showed that median survival was significantly shorter for patients with unsustained CR at 100 days $(n=29)$ compared with those with a lesser response sustained for at least 100 days $(n=667)$, and those with a sustained CR $(n=241)-39$ months, 76 months and not reached, respectively $(P<0.001){ }^{41}$

As mentioned earlier, a number of caveats of these studies should be considered. Survival outcomes can be confounded by 


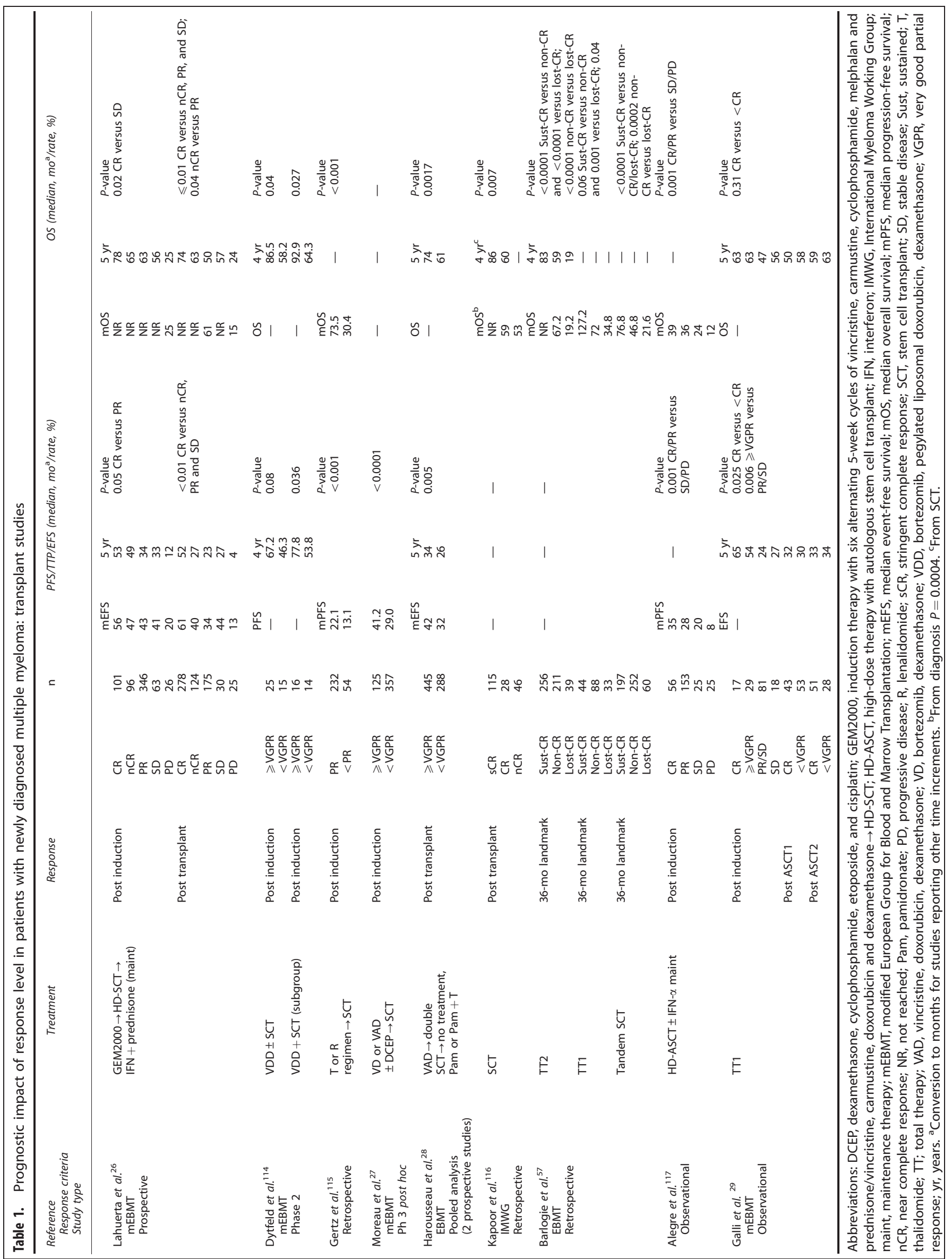


the use of subsequent treatments, including maintenance and salvage therapies with targeted therapies. In the IFM 2005-01 study, patients who achieved at least a PR could enroll onto the IFM 2005-02 study of maintenance therapy with lenalidomide. ${ }^{27}$ Also, the relationship between quality of response and outcomes may be confounded by tumor biology. ${ }^{53}$ In the pooled analysis of GEM2000 and GEM2005 studies, high-risk cytogenetics was an independent predictor of unsustained $C{ }^{41}$ On the other hand, subgroup analyses of the IFM 2005-01 study demonstrated that a quality response improved outcomes in high-risk patients. The median PFS was significantly longer in patients with International Staging System (ISS) 2/3 disease who achieved a post-induction VGPR versus those who did not (not reached versus 23 months; $P<0.0001$ ), as well as in patients with high-risk cytogenetics (37 versus 24 months, respectively, $P=0.0036$ ).

Without a trial specifically designed to assess the quality of response, tumor biology remains a confounding factor. However, more patients, including patients with high-risk MM, are achieving quality responses with targeted therapy regimens compared with conventional chemotherapy, and there is compelling evidence that achieving a quality response can at least partially overcome the impact of negative prognostic factors, including cytogenetic abnormalities. This is particularly evident in the more recent nontransplant studies where HD-ASCT is not an option, and aggressive treatment with targeted therapies has been shown to improve the quality of response and outcomes in high-risk patients. These studies have generally demonstrated important associations between the quality of response and outcomes (Table 2), ${ }^{9,31,43,58-63}$ and pooled analyses have allowed for more robust subgroup analyses to better examine the potential impact of selection bias. ${ }^{30,31}$

Gay et $a l^{31}$ conducted a pooled analysis of three phase 3 studies that treated 1175 patients with MP with or without thalidomide (GISMM-2001 ${ }^{17}$ and HOVON $49^{64}$ studies) and/or bortezomib (GIMEMA-MM-03-05 ${ }^{65}$ ). The highest $C R$ rate was observed in patients receiving VMPT plus VT maintenance (49\%) with the lowest rate in patients receiving MP (5\%), with a robust association between depth of response and outcomes. The 3-year OS rate was $91 \%$ for CR compared with $70 \%$ for VGPR $(P<0.001)$, and $67 \%$ for PR $(P<0.001)$. These relationships were maintained in subgroups older and younger than 75 years of age, and after adjustment for other prognostic factors with multivariate analysis. The time to achieve a CR did not have a significant impact on the outcomes.

More recently, Gay et al. ${ }^{30}$ conducted a separate pooled analysis of 771 patients enrolled in the GIMEMA-MM-03-05 05 and GEM05MAS65 ${ }^{21}$ studies, which assessed the combinations of bortezomib with an IMiD for induction and the use of bortezomib regimens for maintenance therapy. In response-evaluable patients $(n=751), 36 \%$ achieved a CR, $19 \%$ a VGPR and $32 \%$ a PR. Baseline age, ISS stage and cytogenetics- $t(4 ; 14), t(14 ; 16)$ and del17pwere similar across these response subgroups. In a landmark analysis using a 12-month time point, the OS rate at 3 years was $75 \%$ overall and $94 \%$ in the CR subgroup versus $86 \%$ in the VGPR subgroup (hazard ratio (HR) $0.30,95 \%$ confidence interval $(\mathrm{Cl})$ $0.16-0.52, P<0.001)$. The 3 -year OS rate was superior for standardrisk versus high-risk cytogenetics overall (76 versus $65 \%$; HR 0.65 , $95 \% \mathrm{Cl} 0.47-0.91, P=0.01)$, but in patients with a $\mathrm{CR}$, the rates did not differ (94\% versus $90 \%$, respectively; HR $0.66,95 \% \mathrm{Cl}$

Table 2. Prognostic impact of response level in patients with newly diagnosed multiple myeloma: non-transplant studies

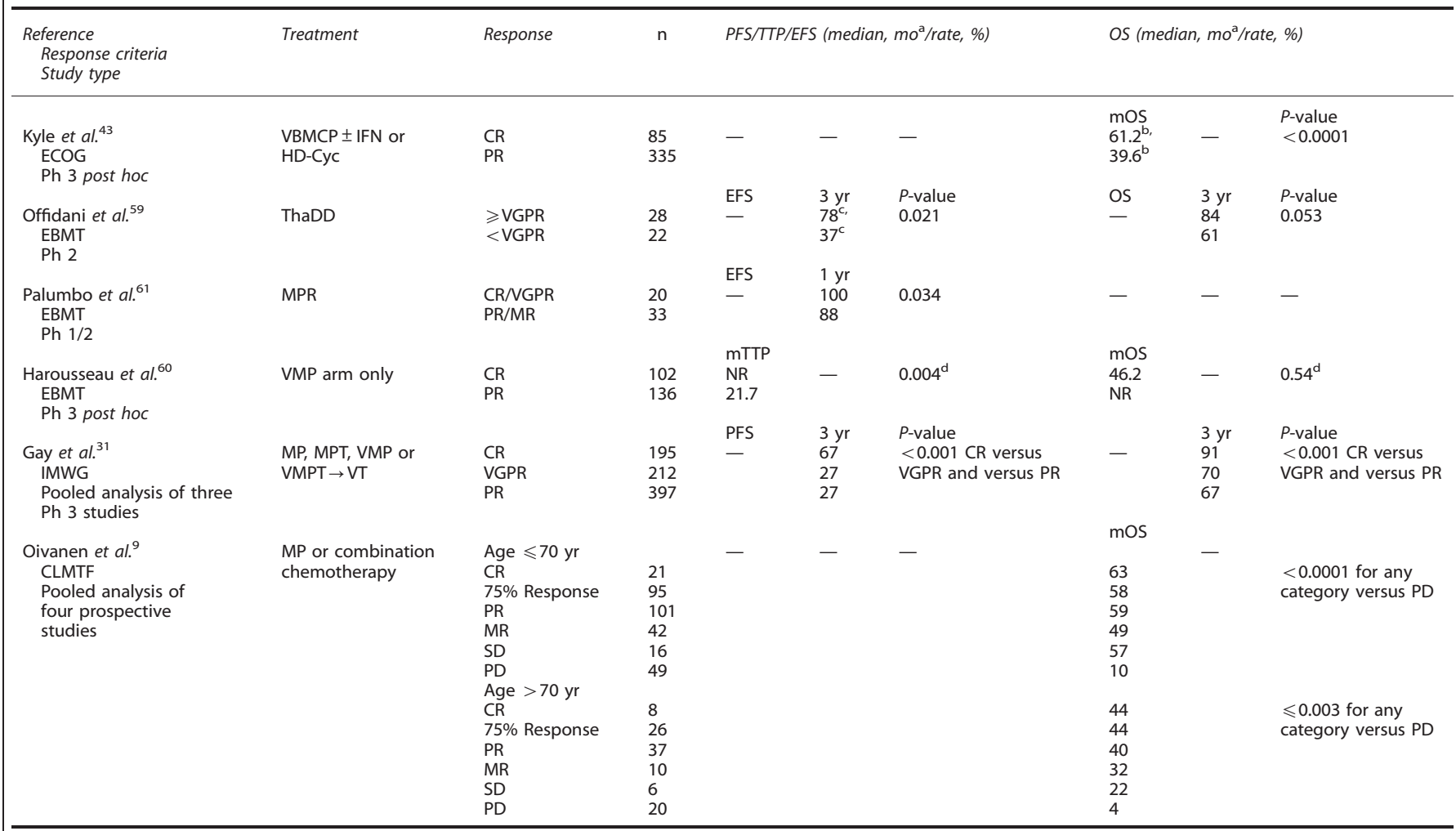

Abbreviations: CLMTF, Chronic Leukemia-Myeloma Task Force; EBMT, European Group for Blood and Marrow Transplantation; ECOG, Eastern Cooperative Oncology Group; EFS, event-free survival; HD, high dose; mOS, median overall survival; MP, melphalan, prednisone; mPFS, median progression-free survival; MPT, melphalan, prednisone, thalidomide; mTTP, median time to progression; MPR, melphalan, prednisone, and lenalidomide; NR, not reached; ThaDD, thalidomide, dexamethasone and pegylated liposomal doxorubicin; VBMCP, vincristine, carmustine (BCNU), melphalan, cyclophosphamide and prednisone;

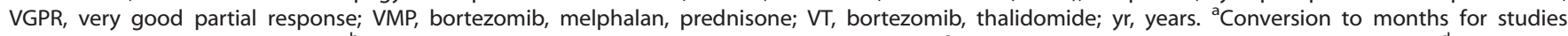
reporting other time increments. ${ }^{b}$ From time of response, converted from years to months. ${ }^{\mathrm{C}}$ Landmark analysis after 4 months of treatment. ${ }^{\mathrm{d}}$ Multivariate analysis. 
0.29-1.50, $P=0.32$ ). A similar trend was seen in a subgroup analysis by ISS stage-significant differences between the ISS stages when considering all evaluable patients, but no difference for patients with a CR. The prognostic significance of $C R$ versus VGPR was maintained following multivariate analysis (HR 0.24, $95 \% \mathrm{Cl} 0.10-0.55, P<0.001)$. Thus, quality of response was a more robust predictor of survival than cytogenetics or ISS stage. Nonetheless, the potential confounding impact of salvage therapies cannot be ruled out, although in maintenance therapy trials variations in treatment after induction can be better controlled because of long-term treatment protocols. Improvement in OS in the context of maintenance trials represents the highest level of evidence achievable in clinical practice.

\section{Relapsed and/or refractory}

In the relapsed or refractory setting, studies have also shown associations between depth of response and outcomes, although data are limited (Table 3). ${ }^{32,58,66-71}$ In the phase 3 APEX study, bortezomib was superior to high-dose dexamethasone for patients with relapsed $\mathrm{MM}$ with respect to response results, time to progression and $\mathrm{OS}^{14}$ An exploratory analysis of the bortezomib arm $(n=315)$ showed no statistical difference in median TTP among patients who achieved a CR (9.7 months) compared with a VGPR (10.8 months) or a PR (8.5 months), whereas CR was associated with significant improvements in the median treatment-free interval (24.1 months) compared with VGPR (6.9 months, $P=0.007)$ and PR (6.4 months, $P=0.002){ }^{66}$ This was likely due in part to the design of the study-once patients achieved a CR, they continued treatment for only two additional cycles. Finally, patients who achieved an MR had a significantly longer TTP compared with non-responders (4.9 versus 2.8 months, $P=0.016)$ and there was a trend of improved OS (24.9 versus 18.7 months).

Other studies have also demonstrated the benefit of achieving an MR in the relapsed/refractory setting. A pooled analysis of two phase 2 studies using thalidomide-containing regimens in patients with relapsed MM showed an association between the level of response and OS with a median OS of $>70$ months for achievement of $\geqslant$ VGPR, 35 months for PR and 11.7 months for MR or non-response $(P<0.001) .{ }^{69}$ MR was favored over nonresponse for PFS (6.1 versus 3.8 months) but not for OS (11 versus 14 months). In a phase 2 study of carfilzomib in patients with relapsed and refractory MM $(n=266)$, median PFS was 10.6 months for patients achieving $\geqslant \operatorname{VGPR}(n=14), 8.3$ months for PR $(n=47)$ and 9.6 months for MR $(n=34)$, whereas the median for the response-evaluable population overall $(n=257)$ was 3.7 months. $^{72}$

\section{IMPROVING THE QUALITY OF RESPONSE AND OUTCOMES}

Despite positive trends in treatment outcomes over the past decade, it has become apparent that treatment strategies need to be further developed to improve the depth and durability of response for the individual patient with $M M$, particularly those with high-risk disease, where the risk of increased toxicity with prolonged or more aggressive treatment is outweighed by the potential survival benefit. ${ }^{73}$ Patients with high-risk cytogenetic abnormalities generally have a shorter duration of response, PFS and OS than standard-risk patients, but the benefit of a CR can be more pronounced. ${ }^{11,74}$ Given that $25-30 \%$ of the NDMM patients present with high-risk disease, ${ }^{75}$ efforts have been made to improve the quality of response and outcomes in these patients, including maintenance therapy and combinations of targeted therapies.

\section{Maintenance therapy}

A number of studies have investigated the efficacy and safety of maintenance treatment with targeted therapies. ${ }^{76-82}$ Studies have consistently demonstrated that thalidomide maintenance after HD-ASCT improves the quality of response and PFS, and some

Table 3. Prognostic impact of response level in patients with relapsed or refractory multiple myeloma

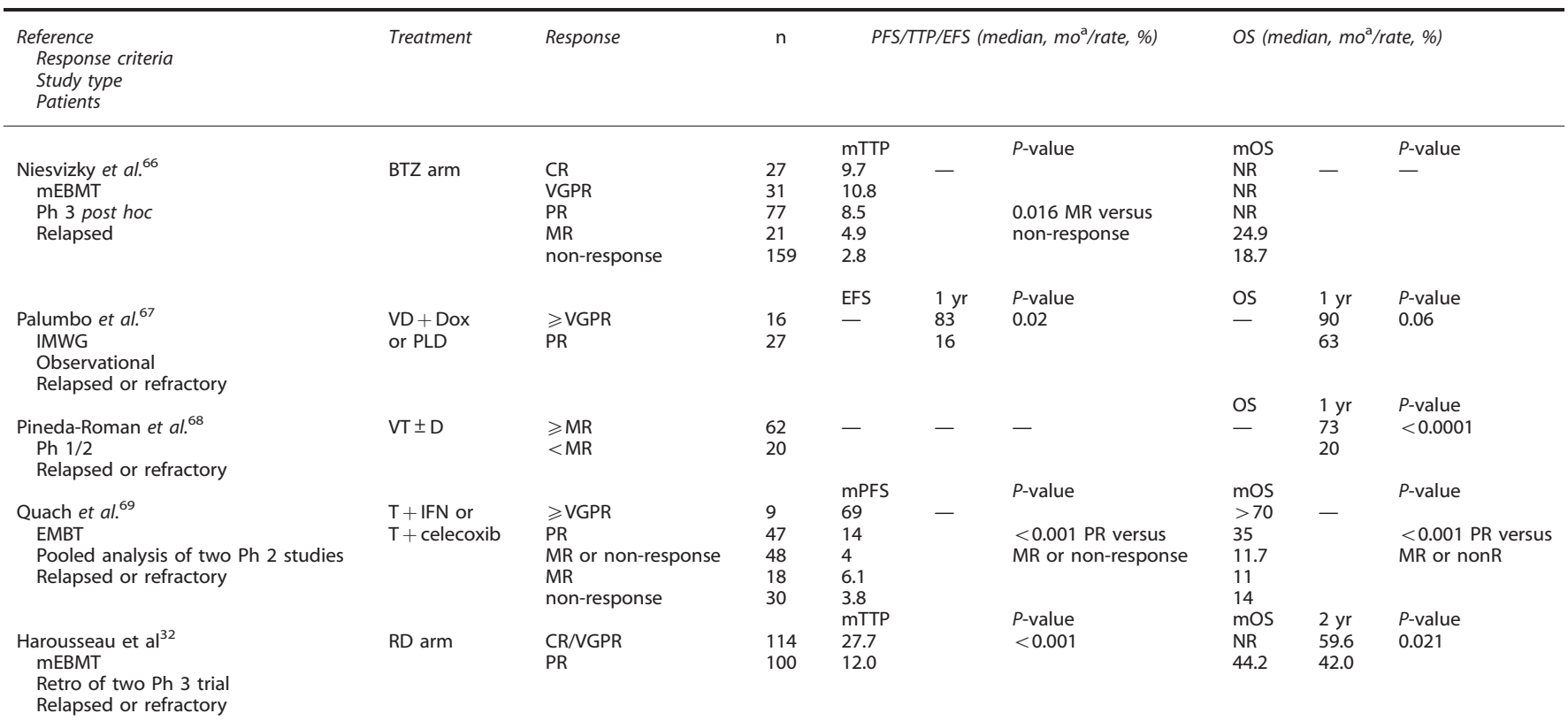

Abbreviations: BTZ, bortezomib; EFS, event-free survival; IFN, interferon; IMWG, International Myeloma Working Group; mEBMT, modified European Group for Blood and Marrow Transplantation; mo, months; mOS, median overall survival; mPFS, median progression-free survival; mTTP, median time to progression; NR, not reached; nonR, non-response; RD, lenalidomide and dexamethasone; T, thalidomide; VD, bortezomib, dexamethasone; VGPR, very good partial response; yr, years. ${ }^{\mathrm{a} C o n v e r s i o n}$ to months for studies reporting other time increments. 
studies have also shown an OS benefit. ${ }^{83}$ However, there is concern about cumulative neurotoxicity with thalidomide.

Lenalidomide maintenance has been assessed in three randomized controlled trials. ${ }^{76-78}$ In one phase 3 trial, 460 patients who had achieved at least stable disease with HD-ASCT were randomized to maintenance with lenalidomide or placebo. ${ }^{77}$ Lenalidomide maintenance was associated with significantly improved TTP compared with placebo (46 versus 27 months; $P<0.001)$ and OS with a 3-year rate of $88 \%$ versus $80 \%(P=0.03)$. This benefit was notable in patients who had not achieved $C R$ with HD-ASCT. In patients who achieved CR with HD-ASCT, the median TTP was not reached in the lenalidomide maintenance arm versus 36 months in the placebo arm (natural log $\mathrm{HR} \mathrm{0.53,95 \%} \mathrm{Cl}$ $-0.001-1.1$ ), with corresponding values of 43 versus 23 months in the non-CR subgroup (natural log HR 0.86, 95\% Cl 0.53-1.2; $P$ for interaction $=0.38$ ). Lenalidomide maintenance was also associated with improved OS in both subgroups, and again the benefit was more pronounced in the non-CR subgroup (natural $\log$ HR 0.53,95\% Cl 0.05-1.0; $P$ for interaction $=0.64$.) than in the CR group (natural log HR $0.25,95 \% \mathrm{Cl}-0.67-1.2$ ). Lenalidomide maintenance was associated with increased toxicity, including grade $3 / 4$ hematologic events (48\% versus $17 \%$, respectively, $P<0.001)$ and secondary primary malignancies (8\% versus $3 \%$, respectively; $P=0.008$ ). In the other two studies, lenalidomide maintenance improved PFS, particularly among transplant-eligible patients who achieved $<$ VGPR with $\mathrm{HD}^{-A S C T^{78}}$ and transplantineligible patients $<75$ years of age. ${ }^{76}$ There was no improvement in OS in either of these studies, but the investigators from both studies noted the need for longer follow-up.

Bortezomib may also be beneficial as a maintenance therapy. ${ }^{80-82,84}$ Sonneveld et al. ${ }^{81}$ recently reported results of a phase 3 study in 827 patients with NDMM who were randomized to induction with VAD or bortezomib, doxorubicin and dexamethasone, followed by HD-ASCT and maintenance with thalidomide for VAD and bortezomib for bortezomib, doxorubicin and dexamethasone. The CR rate overall was $24 \%$ for the VAD arm versus $36 \%$ for the bortezomib, doxorubicin and dexamethasone arm $(P<0.001)$, the rate of improved response during maintenance treatment was $24 \%$ and $23 \%(P=0.64)$, respectively. Median PFS was 28 versus 35 months, respectively $(P=0.002)$, and median OS had not been reached in either arm. Subgroup analysis of patients with the $17 p 13$ deletion showed a $\geqslant n C R$ rate of $20 \%$ in the VAD arm versus $52 \%$ in the bortezomib, doxorubicin and dexamethasone arm $(P=0.008)$, which corresponded to median os of 24 versus $>54$ months (HR 0.36, 95\% Cl 0.18-0.74; $P=0.003$ ). In the subgroup without the 17p13 deletion, there was no difference in OS (HR 0.96, 95\% Cl 0.69-1.34; $P=0.81$ ).

As data for maintenance therapy accumulate, it will be important to assess the impact quality of response has on outcomes in different risk groups. Although there is compelling evidence that driving response deeper with maintenance therapy in standard-risk patients improves PFS and possibly OS, ${ }^{26,55}$ PFS is not a suitable surrogate for OS in this patient population. ${ }^{15,78,85,86}$ Without prolonged follow-up and OS data, it is unclear if those achieving CR after HD-ASCT should receive maintenance treatment or wait until relapse. In high-risk patients where $C R$ has been shown to be an important predictor of outcome, PFS is a suitable primary endpoint as it usually correlates with OS. ${ }^{11,30,87}$ For high-risk patients, it is not so much a question of maintenance therapy but rather if a multidrug combination is appropriate. $^{80,83,84,87}$

Multidrug treatment strategies-combining Pls and immunomodulatory drugs

Pairing Pls with IMiDs or cytotoxic agents, usually in triplet combinations with a corticosteroid, is changing the treatment landscape in MM as factors associated with poor outcomes appear to have far less impact. ${ }^{88}$ The rationale for using these combinations is supported by the preclinical data demonstrating that IMiDs potentiate the activity of PIs and dexamethasone ${ }^{89-92}$ and of synergistic activity between PIs and cytotoxic agents. ${ }^{93-95}$ Many of the toxicities associated with these drug classes are non-overlapping. So far, triplet combinations with a PI, IMiD and corticosteroid have demonstrated exceptional responses in both the frontline and salvage settings, and in patients with high-risk disease (Table 4). ${ }^{19-24,96-99}$ Generally, these combinations have been well tolerated, as high anti-MM activity has allowed the development of regimens with reduced doses or different dosing schedules of the individual agents. ${ }^{20,22,24,98}$ Nonetheless, there are increases in some toxicities, and there may be limits to the combinations currently available. In the phase 2 EVOLUTION study, for example, a quadruplet combination of bortezomib, dexamethasone, cyclophosphamide and lenalidomide was highly active in NDMM but did not provide a clinical benefit over bortezomib-based triplet combinations and was associated with greater toxicity. ${ }^{100}$ Other than the pooled analysis of the GIMEMAMM-03-05 and GEM05MAS65 studies discussed earlier, ${ }^{30}$ the impact of quality of response on outcomes has not been well studied with these combination regimens. ${ }^{19,30}$

\section{REDEFINING CR-IMMUNOPHENOTYPIC AND MOLECULAR CRS}

As the rates of $C R$ and stringent $C R$ continue to advance, it is of interest to further stratify the CR category. Assessment of minimal residual disease (MRD) in bone marrow plasma cells with sensitive assays that define immunophenotypic and molecular responses is becoming a useful measure of response with potential prognostic implications. ${ }^{21,56,101-105}$ Immunophenotypic response is assessed by multiparametric flow cytometry, which uses immunofluorescence with monoclonal antibodies to plasma cell proteins (for example, CD38, CD19 and CD117) to identify, quantify and characterize bone marrow plasma cell composition. ${ }^{101-103}$ Molecular response is assessed with allelespecific oligonucleotide PCR. Tumor-specific primers are constructed for individual patients from the rearranged variable region of immunoglobulin heavy-chain genes through reverse transcription of RNA (extracted from bone marrow plasma cells) and generation of the immunoglobulin heavy-chain genes complementary DNA. ${ }^{56,106}$

In a prospective analysis of 295 patients with NDMM uniformly treated with the GEM2000 protocol, the immunophenotypic response was assessed by the multiparametric flow cytometry method (sensitivity $10^{-4}$ ) at 100 days post transplantation. ${ }^{103}$ At day 100, 147 patients had achieved a CR, with 58\% being MRD positive and $42 \%$ MRD negative. In the MRD-positive group, the proportion of abnormal plasma cells corresponded to depth of response, with a mean of $0.10 \%$ for $C R, 0.21 \%$ for $n C R$ and $0.76 \%$ for PR $(P=0.001)$. PFS was significantly longer for MRD-negative versus MRD-positive patients (median, 71 versus 37 months, $P<0.001)$, as was OS (5-year rate of 82 versus $60 \%, P=0.002$ ), with the benefit independent of immunofixation status. For the immunofixation-negative subgroup (that is, CR), the 5-year PFS rate was $62 \%$ in MRD-negative versus $30 \%$ in MRD-positive patients $(P<0.001)$ and the 5 -year OS rate was $87 \%$ versus $59 \%$, respectively $(P=0.009)$.

Ladetto et al. $^{56}$ assessed MRD by the nested-PCR method (sensitivity $10^{-6}$ ) in 39 patients with NDMM who achieved a $\geqslant$ VGPR with HD-ASCT received consolidation therapy with bortezomib, thalidomide, dexamethasone. At study entry, 33 patients $(85 \%)$ had a VGPR following HD-ASCT, 6 had a CR (15\%) with $1(3 \%)$ a molecular CR. All patients received one course of bortezomib, thalidomide, dexamethasone and 31 completed four courses. After consolidation, the CR rate improved to $49 \%$ and molecular CR improved to $18 \%$. After a median follow-up of 42 
Table 4. Clinical studies in patients with multiple myeloma receiving combination regimens that include a proteasome inhibitor and an IMiD

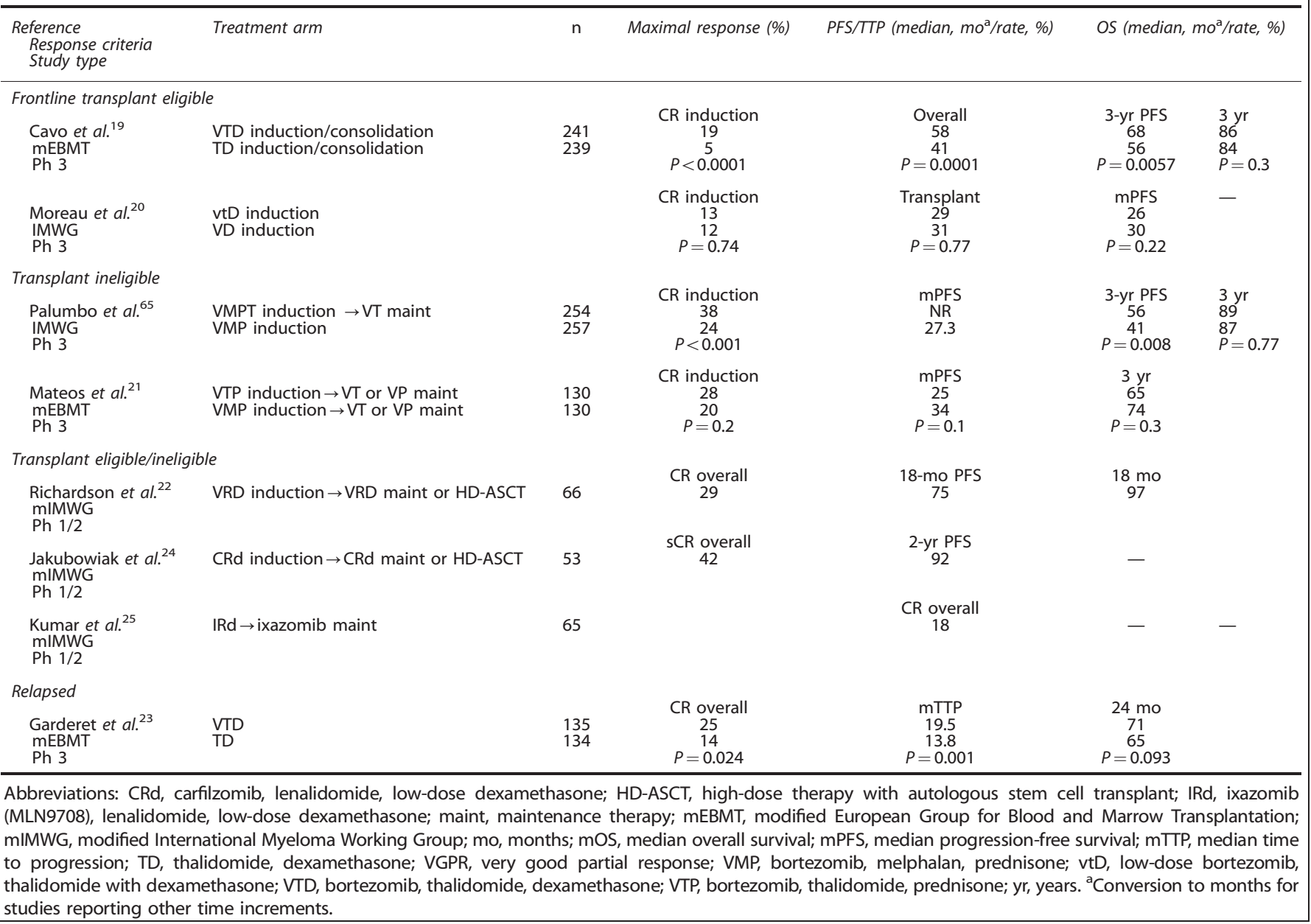

months, all patients who achieved a molecular response were relapse-free, whereas 11 patients who were MRD-positive had relapsed.

These new assays will be critical in determining the benefit of aggressive and prolonged therapies, and are potential surrogate markers for OS. In 2011, the International Myeloma Working Group updated the response criteria to include immunophenotypic $C R$, defined as a stringent $C R$ with evidence of MRD-negative disease by multiparametric flow cytometry (absence of phenotypically aberrant clonal plasma cells in $\geqslant 1$ million analyzed cells), and molecular CR, a CR with evidence of MRD-negative disease by the PCR method (sensitivity $10^{-5}$ ). ${ }^{107}$ Although a number of studies are now using MRD as a study endpoint, ${ }^{21,24,100,108}$ these assays are complex, not applicable to all patients and allele-specific oligonucleotide PCR can be expensive and time consuming, so their wider adoption may take time.

\section{FUTURE CONSIDERATIONS}

The development of targeted therapies for MM has not only resulted in clinically significant improvements in outcomes but has also advanced our understanding of this complex disease. Collectively, the results from studies of targeted therapies support maximizing response at all treatment stages and in most patient subgroups. However, these are not definitive data and there remain important gaps in our knowledge. We need to more accurately predict the individual response level that is durable and will extend disease control and survival without significant risk of treatment-related morbidity. Some patients can achieve long-term survival with a lesser response, but this may or may not require continuous treatment, and the potential benefit of treating a patient to a deeper response with more aggressive treatment of shorter duration should also be weighed. Treatment decisions-initiating a new treatment or intensifying the current treatment-need to be better informed across the levels of reponse. $^{10}$ This will require a greater understanding of the molecular evolution of MM over the course of the disease and treatment, to identify and differentiate high- to low-risk subclones and their sensitivity to the selective pressures of specific treatments at initial diagnosis and disease progression. ${ }^{33-35}$ This could lead to strategies that primarily target the highly proliferative and genetically dynamic subclones with aggressive multi-agent treatment, and secondarily target indolent and genetically stable subclones with more selective regimens but this will require further study in the clinical setting. Finally, the cost of targeted therapies-particularly, prolonged treatment (for example, maintenance or salvage therapy) - is an important barrier that should be acknowledged, as some healthcare systems may not provide support for their use in certain settings without definitive data.

In the transplant setting, the development of triplet combinations pairing Pls and IMiDs has improved the depth of response with induction therapy. Triplet combinations allow for more rapid and high-level responses, and may overwhelm the potential resistance through synergistic and complementary antimyeloma activity. ${ }^{88}$ The quality of response with some triplet combinations has reached a level where it might be better to defer HD-ASCT until relapse or use it strategically to improve response. ${ }^{109}$ 
Ongoing studies should help to address these issues (ClinicalTrials.gov NCT01191060 and NCT01208766).

Although tolerability with multidrug regimens is always a concern, various treatment strategies (for example, attenuated dosing $^{5}$ ) are available to address these in specific patients and will help to broaden the spectrum of patients considered for multidrug regimens. Furthermore, novel Pls (for example, carfilzomib and ixazomib [MLN9708]) and IMiDs (for example, pomalidomide) have demonstrated good clinical activity and are well tolerated, ${ }^{24,25,72,98,99,110,111}$ and other classes of targeted therapy (for example, histone deacetylase inhibitors and monoclonal antibodies) have shown encouraging activity. ${ }^{112,113}$ With more options, treatment regimens can be better tailored to the individual patient.

The development of laboratory and animal models of myeloma and the bone marrow microenvironment in the 1990s proved instrumental in rapidly bringing the targeted therapies from the bench to the bedside and in the development of combination regimens. ${ }^{18}$ Moving forward, preclinical models will help us to better understand the mechanisms underlying the quality of responses, to develop biomarkers that predict treatment response and outcomes, and to maximize the potential of targeted therapies. It is equally important to continue to develop response assays that can further differentiate the impact of high-level response and are accessible to a range of institutions.

\section{CONCLUSIONS}

In the era of targeted therapies, quality of response is associated with disease control and survival in patients with $\mathrm{MM}$, including patients with high-risk disease, but until clinical trials are specifically designed to assess this relationship, it will remain only an association. Achievement of maximal response should be considered upfront and at all stages of treatment, bearing in mind tolerability, quality of life and treatment barriers including cost and the lack of definitive data. In select patients, achievement of a lesser response may be adequate to prolong survival and attempts to treat these patients to a better response may place them at unnecessary risk without a significant benefit. Multidrug regimens combining PIs with IMiDs have improved depth of response, have acceptable tolerability and are becoming a standard treatment approach. The development of novel targeted therapies should further advance these goals as clinical data in conjunction with laboratory findings should help to facilitate the use of antimyeloma agents to their fullest potential. Treatment should be tailored to the disease characteristics and needs and goals of the individual patient.

\section{CONFLICT OF INTEREST}

Dr Lonial has received compensation as a consultant for Millennium, Celgene, Novartis, Bristol-Myers Squibb, Onyx Pharmaceuticals and Merck. Dr Anderson has received compensation as a member of the scientific advisory boards of Celgene, Onyx, Gilead, and Sanofi-Aventis. He is also a scientific founder of Acetylon and Oncopep.

\section{ACKNOWLEDGEMENTS}

Dr Lonial's position is funded, in part, by the 'Ray C. Osborne Fund' at Emory University School of Medicine. We thank Michael Raffin (Fishawack Communications) for medical writing and editorial assistance, which was supported by Onyx Pharmaceuticals, Inc.

\section{REFERENCES}

1 Kumar SK, Rajkumar SV, Dispenzieri A, Lacy MQ, Hayman SR, Buadi FK et al. Improved survival in multiple myeloma and the impact of novel therapies. Blood 2008; 111: 2516-2520.
2 Pulte D, Gondos A, Brenner H. Improvement in survival of older adults with multiple myeloma: results of an updated period analysis of SEER data. Oncologist 2011; 16: 1600-1603.

3 Brenner H, Gondos A, Pulte D. Expected long-term survival of patients diagnosed with multiple myeloma in 2006-2010. Haematologica 2009; 94 : 270-275.

4 Kumar S. Treatment of newly diagnosed multiple myeloma in transplant-eligible patients. Curr Hematol Malig Rep 2011; 6: 104-112.

5 Palumbo A, Anderson K. Multiple myeloma. N Engl J Med 2011; 364: 1046-1060.

6 Kumar S. Multiple myeloma-current issues and controversies. Cancer Treat Rev 2010; 36(Suppl 2): S3-S11.

7 Davies FE, Forsyth PD, Rawstron AC, Owen RG, Pratt G, Evans PA et al. The impact of attaining a minimal disease state after high-dose melphalan and autologous transplantation for multiple myeloma. Br J Haematol 2001; 112: 814-819.

8 Blade J, Lopez-Guillermo A, Bosch F, Cervantes F, Reverter JC, Montserrat E et al. Impact of response to treatment on survival in multiple myeloma: results in a series of 243 patients. Br J Haematol 1994; 88: 117-121.

9 Oivanen TM, Kellokumpu-Lehtinen P, Koivisto AM, Koivunen E, Palva I. Response level and survival after conventional chemotherapy for multiple myeloma: a Finnish Leukaemia Group study. Eur J Haematol 1999; 62: 109-116.

10 Chanan-Khan AA, Giralt S. Importance of achieving a complete response in multiple myeloma, and the impact of novel agents. J Clin Oncol 2010; 28: 2612-2624.

11 Haessler J, Shaughnessy Jr. JD, Zhan F, Crowley J, Epstein J, van Rhee F et al. Benefit of complete response in multiple myeloma limited to high-risk subgroup identified by gene expression profiling. Clin Cancer Res 2007; 13: 7073-7079.

12 Dimopoulos M, Spencer A, Attal M, Prince HM, Harousseau JL, Dmoszynska A et al. Lenalidomide plus dexamethasone for relapsed or refractory multiple myeloma. N Engl J Med 2007; 357: 2123-2132.

13 Weber DM, Chen C, Niesvizky R, Wang M, Belch A, Stadtmauer EA et al. Lenalidomide plus dexamethasone for relapsed multiple myeloma in North America. N Engl J Med 2007; 357: 2133-2142.

14 Richardson PG, Sonneveld P, Schuster MW, Irwin D, Stadtmauer EA, Facon T et al. Bortezomib or high-dose dexamethasone for relapsed multiple myeloma. $N$ Engl J Med 2005; 352: 2487-2498.

15 Barlogie B, Tricot G, Anaissie E, Shaughnessy J, Rasmussen E, van Rhee F et al. Thalidomide and hematopoietic-cell transplantation for multiple myeloma. N Engl J Med 2006; 354: 1021-1030.

16 San Miguel JF, Schlag R, Khuageva NK, Dimopoulos MA, Shpilberg O, Kropff M et al. Bortezomib plus melphalan and prednisone for initial treatment of multiple myeloma. N Engl J Med 2008; 359: 906-917.

17 Palumbo A, Bringhen S, Caravita T, Merla E, Capparella V, Callea V et al. Oral melphalan and prednisone chemotherapy plus thalidomide compared with melphalan and prednisone alone in elderly patients with multiple myeloma: randomised controlled trial. Lancet 2006; 367: 825-831.

18 Anderson KC. The 39th David A. Karnofsky Lecture: bench-to-bedside translation of targeted therapies in multiple myeloma. J Clin Oncol 2012; 30: 445-452.

19 Cavo M, Tacchetti P, Patriarca F, Petrucci MT, Pantani L, Galli M et al. Bortezomib with thalidomide plus dexamethasone compared with thalidomide plus dexamethasone as induction therapy before, and consolidation therapy after, double autologous stem-cell transplantation in newly diagnosed multiple myeloma: a randomised phase 3 study. Lancet 2010; 376: 2075-2085.

20 Moreau P, Avet-Loiseau H, Facon T, Attal M, Tiab M, Hulin C et al. Bortezomib plus dexamethasone versus reduced-dose bortezomib, thalidomide plus dexamethasone as induction treatment before autologous stem cell transplantation in newly diagnosed multiple myeloma. Blood 2011; 118: 5752-5758

21 Mateos MV, Oriol A, Martinez-Lopez J, Gutierrez N, Teruel Al, de Paz R et al. Bortezomib, melphalan, and prednisone versus bortezomib, thalidomide, and prednisone as induction therapy followed by maintenance treatment with bortezomib and thalidomide versus bortezomib and prednisone in elderly patients with untreated multiple myeloma: a randomised trial. Lancet Oncol 2010; 11: 934-941.

22 Richardson PG, Weller E, Lonial S, Jakubowiak AJ, Jagannath S, Raje NS et al. Lenalidomide, bortezomib, and dexamethasone combination therapy in patients with newly diagnosed multiple myeloma. Blood 2010; 116: 679-686.

23 Garderet L, lacobelli S, Moreau P, Dib M, Lafon I, Niederwieser D et al. Superiority of the triple combination of bortezomib-thalidomide-dexamethasone over the dual combination of thalidomide-dexamethasone in patients with multiple myeloma progressing or relapsing after autologous transplantation: the MMVAR/IFM 2005-04 Randomized Phase III Trial From the Chronic Leukemia Working Party of the European Group for Blood and Marrow Transplantation. J Clin Oncol 2012; 30: 2475-2482. 
24 Jakubowiak AJ, Dytfeld D, Griffith KA, Lebovic D, Vesole DH, Jagannath S et al. A phase $1 / 2$ study of carfilzomib in combination with lenalidomide and low-dose dexamethasone as a frontline treatment for multiple myeloma. Blood 2012; 120: 1801-1809.

25 Kumar SK, Berdeja JG, Niesvizky R, Lonial S, Hamadani M, Stewart AK et al. A phase $1 / 2$ study of weekly MLN9708, an investigational oral proteasome inhibitor, in combination with lenalidomide and dexamethasone in patients with previously untreated multiple myeloma (MM). ASH Annual Meeting Abstracts 2012; 120: 332.

26 Lahuerta JJ, Mateos MV, Martinez-Lopez J, Rosinol L, Sureda A, de la Rubia J et al. Influence of pre- and post-transplantation responses on outcome of patients with multiple myeloma: sequential improvement of response and achievement of complete response are associated with longer survival. J Clin Oncol 2008; 26: 5775-5782.

27 Moreau P, Attal M, Pegourie B, Planche L, Hulin C, Facon T et al. Achievement of VGPR to induction therapy is an important prognostic factor for longer PFS in the IFM 2005-01 trial. Blood 2011; 117: 3041-3044.

28 Harousseau JL, Avet-Loiseau H, Attal M, Charbonnel C, Garban F, Hulin C et al. Achievement of at least very good partial response is a simple and robust prognostic factor in patients with multiple myeloma treated with high-dose therapy: long-term analysis of the IFM 99-02 and 99-04 Trials. J Clin Oncol 2009; 27: $5720-5726$.

29 Galli M, Nicolucci A, Valentini M, Belfiglio M, Delaini F, Crippa C et al. Feasibility and outcome of tandem stem cell autotransplants in multiple myeloma. Haematologica 2005; 90: 1643-1649.

30 Gay F, Larocca A, Mateos M, Oliva S, Offidani M, Ria R et al. Complete response is a stronger predictor of survival than cytogenetic profile and International Staging System stage in elderly myeloma patients treated with bortezomib: analysis of 771 patients. EHA 2012 Haematologica 97(supp 1); abstract O0591.

31 Gay F, Larocca A, Wijermans P, Cavallo F, Rossi D, Schaafsma R et al. Complete response correlates with long-term progression-free and overall survival in elderly myeloma treated with novel agents: analysis of 1175 patients. Blood 2011; 117: 3025-3031.

32 Harousseau JL, Dimopoulos MA, Wang M, Corso A, Chen C, Attal M et al. Better quality of response to lenalidomide plus dexamethasone is associated with improved clinical outcomes in patients with relapsed or refractory multiple myeloma. Haematologica 2010; 95: 1738-1744.

33 Egan JB, Shi CX, Tembe W, Christoforides A, Kurdoglu A, Sinari S et al. Whole genome sequencing of multiple myeloma from diagnosis to plasma cell leukemia reveals genomic initiating events, evolution and clonal tides. Blood 2012; 120: 1060-1066.

34 Keats JJ, Chesi M, Egan JB, Garbitt VM, Palmer SE, Braggio E et al. Clonal competition with alternating dominance in multiple myeloma. Blood 2012; 120 1067-1076.

35 Morgan GJ, Walker BA, Davies FE. The genetic architecture of multiple myeloma Nat Rev Cancer 2012; 12: 335-348.

36 Harousseau JL, Attal M, Avet-Loiseau H. The role of complete response in multiple myeloma. Blood 2009; 114: 3139-3146.

37 Rajkumar SV, Gahrton G, Bergsagel PL. Approach to the treatment of multiple myeloma: a clash of philosophies. Blood 2011; 118: 3205-3211.

38 Anderson KC, Kyle RA, Rajkumar SV, Stewart AK, Weber D, Richardson P. Clinically relevant end points and new drug approvals for myeloma. Leukemia 2008; 22: 231-239.

39 Pineda-Roman M, Bolejack V, Arzoumanian V, Anaissie E, van Rhee F, Zangari M et al. Complete response in myeloma extends survival without, but not with history of prior monoclonal gammopathy of undetermined significance or smouldering disease. Br J Haematol 2007; 136: 393-399.

40 Zhan F, Barlogie B, Arzoumanian V, Huang Y, Williams DR, Hollmig K et al. Gene-expression signature of benign monoclonal gammopathy evident in multiple myeloma is linked to good prognosis. Blood 2007; 109: 1692-1700.

41 Paiva B, Gutierrez NC, Rosinol L, Vidriales MB, Montalban MA, Martinez-Lopez J et al. High-risk cytogenetics and persistent minimal residual disease by multiparameter flow cytometry predict unsustained complete response after autologous stem cell transplantation in multiple myeloma. Blood 2012; 119 687-691.

42 Riccardi A, Mora O, Tinelli C, Porta C, Danova M, Brugnatelli S et al. Response to first-line chemotherapy and long-term survival in patients with multiple myeloma: results of the MM87 prospective randomised protocol. Eur J Cancer 2003; 39: 31-37.

43 Kyle RA, Leong T, Li S, Oken MM, Kay NE, Van Ness B et al. Complete response in multiple myeloma: clinical trial E9486, an Eastern Cooperative Oncology Group study not involving stem cell transplantation. Cancer 2006; 106: 1958-1966.
44 Blade J, Samson D, Reece D, Apperley J, Bjorkstrand B, Gahrton G et al. Criteria for evaluating disease response and progression in patients with multiple myeloma treated by high-dose therapy and haemopoietic stem cell transplantation. Myeloma Subcommittee of the EBMT. European Group for Blood and Marrow Transplant. Br J Haematol 1998; 102: 1115-1123.

45 Durie BG, Harousseau JL, Miguel JS, Blade J, Barlogie B, Anderson K et al. International uniform response criteria for multiple myeloma. Leukemia 2006; 20 : 1467-1473.

46 Richardson PG, Barlogie B, Berenson J, Singhal S, Jagannath S, Irwin D et al. A phase 2 study of bortezomib in relapsed, refractory myeloma. $N$ Engl J Med 2003; 348: 2609-2617.

47 Krejci M, Scudla V, Tothova E, Schutzova M, Koza V, Adam Z et al. Long-term outcomes of autologous transplantation in multiple myeloma: significant survival benefit of novel drugs in post-transplantation relapse. Clin Lymphoma Myeloma 2009; 9: 436-442.

48 Venner CP, Connors JM, Sutherland HJ, Shepherd JD, Hamata L, Mourad YA et al. Novel agents improve survival of transplant patients with multiple myeloma including those with high-risk disease defined by early relapse ( $<12$ months). Leuk Lymphoma 2011; 52: 34-41.

49 Pazdur R. Endpoints for assessing drug activity in clinical trials. Oncologist 2008; 13(Suppl 2): 19-21.

50 Lenhoff S, Hjorth M, Turesson I, Westin J, Gimsing P, Wisloff F et al. Intensive therapy for multiple myeloma in patients younger than 60 years. Long-term results focusing on the effect of the degree of response on survival and relapse pattern after transplantation. Haematologica 2006; 91: $1228-1233$.

51 Durie BG, Jacobson J, Barlogie B, Crowley J. Magnitude of response with myeloma frontline therapy does not predict outcome: importance of time to progression in southwest oncology group chemotherapy trials. J Clin Oncol 2004; 22: 1857-1863.

52 Singhal S, Powles R, Sirohi B, Treleaven J, Kulkarni S, Mehta J. Response to induction chemotherapy is not essential to obtain survival benefit from high-dose melphalan and autotransplantation in myeloma. Bone Marrow Transplant 2002; 30: 673-679.

53 Rajkumar SV, Fonseca R, Dispenzieri A, Lacy MQ, Witzig TE, Lust JA et al. Effect of complete response on outcome following autologous stem cell transplantation for myeloma. Bone Marrow Transplant 2000; 26: 979-983.

54 Nooka AK, Kaufman JL, Behera M, Gleason C, Langston A, Steuer CE et al. The improved efficacy of bortezomib containing induction regimens $(B C I R)$ versus non-bortezomib containing induction regimens (NBCIR) in transplant-eligible patients with multiple myeloma (MM): meta-analysis of phase III randomized controlled trials (RCTs). Blood 2011; 118: (abstract 3994).

55 Wang M, Delasalle K, Feng L, Thomas S, Giralt S, Qazilbash M et al. CR represents an early index of potential long survival in multiple myeloma. Bone Marrow Transplant 2010; 45: 498-504.

56 Ladetto M, Pagliano G, Ferrero S, Cavallo F, Drandi D, Santo L et al. Major tumor shrinking and persistent molecular remissions after consolidation with bortezomib, thalidomide, and dexamethasone in patients with autografted myeloma. J Clin Oncol 2010; 28: 2077-2084.

57 Barlogie B, Anaissie E, Haessler J, van Rhee F, Pineda-Roman M, Hollmig K et al. Complete remission sustained 3 years from treatment initiation is a powerful surrogate for extended survival in multiple myeloma. Cancer 2008; 113: 355-359.

58 Hussein MA, Baz R, Srkalovic G, Agrawal N, Suppiah R, Hsi E et al. Phase 2 study of pegylated liposomal doxorubicin, vincristine, decreased-frequency dexamethasone, and thalidomide in newly diagnosed and relapsed-refractory multiple myeloma. Mayo Clin Proc 2006; 81: 889-895.

59 Offidani M, Corvatta L, Piersantelli MN, Visani G, Alesiani F, Brunori M et al. Thalidomide, dexamethasone, and pegylated liposomal doxorubicin (ThaDD) for patients older than 65 years with newly diagnosed multiple myeloma. Blood 2006; 108: 2159-2164.

60 Harousseau JL, Palumbo A, Richardson PG, Schlag R, Dimopoulos MA, Shpilberg $\mathrm{O}$ et al. Superior outcomes associated with complete response in newly diagnosed multiple myeloma patients treated with nonintensive therapy: analysis of the phase 3 VISTA study of bortezomib plus melphalan-prednisone versus melphalan-prednisone. Blood 2010; 116: 3743-3750.

61 Palumbo A, Falco P, Corradini P, Falcone A, Di Raimondo F, Giuliani N et al. Melphalan, prednisone, and lenalidomide treatment for newly diagnosed myeloma: a report from the GIMEMA--Italian Multiple Myeloma Network. J Clin Oncol 2007; 25: 4459-4465.

62 Morgan GJ, Davies FE, Gregory WM, Russell NH, Bell SE, Szubert AJ et al. Cyclophosphamide, thalidomide, and dexamethasone (CTD) as initial therapy for patients with multiple myeloma unsuitable for autologous transplantation. Blood 2011; 118: 1231-1238. 
63 Schaar CG, Kluin-Nelemans JC, le Cessie S, Franck PF, te Marvelde MC, Wijermans PW. Early response to therapy and survival in multiple myeloma. $\mathrm{Br} J$ Haematol 2004; 125: 162-166.

64 Wijermans P, Schaafsma M, Termorshuizen F, Ammerlaan R, Wittebol S, Sinnige H et al. Phase III study of the value of thalidomide added to melphalan plus prednisone in elderly patients with newly diagnosed multiple myeloma: the HOVON 49 Study. J Clin Oncol 2010; 28: 3160-3166.

65 Palumbo A, Bringhen S, Rossi D, Cavalli M, Larocca A, Ria R et al. Bortezomib-melphalan-prednisone-thalidomide followed by maintenance with bortezomib-thalidomide compared with bortezomib-melphalan-prednisone for initial treatment of multiple myeloma: a randomized controlled trial. $J$ Clin Oncol 2010; 28: 5101-5109.

66 Niesvizky R, Richardson PG, Rajkumar SV, Coleman M, Rosinol L, Sonneveld P et al. The relationship between quality of response and clinical benefit for patients treated on the bortezomib arm of the international, randomized, phase 3 APEX trial in relapsed multiple myeloma. Br J Haematol 2008; 143 : 46-53.

67 Palumbo A, Gay F, Bringhen S, Falcone A, Pescosta N, Callea V et al. Bortezomib, doxorubicin and dexamethasone in advanced multiple myeloma. Ann Oncol 2008; 19: 1160-1165.

68 Pineda-Roman M, Zangari M, van Rhee F, Anaissie E, Szymonifka J, Hoering A et al. VTD combination therapy with bortezomib-thalidomide-dexamethasone is highly effective in advanced and refractory multiple myeloma. Leukemia 2008; 22: 1419-1427.

69 Quach H, Mileshkin L, Seymour JF, Milner A, Ritchie D, Harrison S et al. Predicting durable remissions following thalidomide therapy for relapsed myeloma. Leuk Lymphoma 2009; 50: 223-229.

70 Alegre A, Aguado B, Giraldo P, Rios E, Canovas A, Ibanez A et al. Lenalidomide is effective as salvage therapy in refractory or relapsed multiple myeloma: analysis of the Spanish Compassionate Use Registry in advanced patients. Int J Hematol 2011; 93: 351-360.

71 Richardson PG, Sonneveld P, Schuster M, Irwin D, Stadtmauer E, Facon T et al. Extended follow-up of a phase 3 trial in relapsed multiple myeloma: final time-to-event results of the APEX trial. Blood 2007; 110: 3557-3560.

72 Siegel DS, Martin T, Wang M, Vij R, Jakubowiak AJ, Lonial S et al. A phase 2 study of single-agent carfilzomib (PX-171-003-A1) in patients with relapsed and refractory multiple myeloma. Blood 2012; 120: 2817-2825.

73 Avet-Loiseau H. Ultra high-risk myeloma. Hematology Am Soc Hematol Educ Program 2010; 2010: 489-493.

74 Rajkumar SV. Multiple myeloma: 2012 update on diagnosis, risk-stratification, and management. Am J Hematol 2012; 87: 78-88.

75 Kyle RA, Rajkumar SV. Criteria for diagnosis, staging, risk stratification and response assessment of multiple myeloma. Leukemia 2009; 23: 3-9.

76 Palumbo A, Hajek R, Delforge M, Kropff M, Petrucci MT, Catalano J et al. Continuous lenalidomide treatment for newly diagnosed multiple myeloma. N Engl J Med 2012; 366: 1759-1769.

77 McCarthy PL, Owzar K, Hofmeister CC, Hurd DD, Hassoun H, Richardson PG et al. Lenalidomide after stem-cell transplantation for multiple myeloma. $N$ Engl J Med 2012; 366: 1770-1781.

78 Attal M, Lauwers-Cances V, Marit G, Caillot D, Moreau P, Facon T et al. Lenalidomide maintenance after stem-cell transplantation for multiple myeloma. N Engl J Med 2012; 366: 1782-1791.

79 Nooka AK, Behera M, Boise LH, Watson M, Kaufman JL, Lonial S. Thalidomide as maintenance therapy in multiple myeloma (MM) improves progression free survival (PFS) and overall survival (OS): a meta-analysis. Blood 2011; 118: (abstract 1855).

80 Mateos MV, Oriol A, Martinez-Lopez J, Gutierrez N, Teruel Al, Lopez de la Guia A et al. Maintenance therapy with bortezomib plus thalidomide or bortezomib plus prednisone in elderly multiple myeloma patients included in the GEM2005MAS65 trial. Blood 2012; 120: 2581-2588.

81 Sonneveld P, Schmidt-Wolf IG, van der Holt B, El Jarari L, Bertsch U, Salwender H et al. Bortezomib induction and maintenance treatment in patients with newly diagnosed multiple myeloma: results of the randomized phase III HOVON-65/ GMMG-HD4 trial. J Clin Oncol 2012; 30: 2946-2955.

82 Niesvizky R, Flinn IW, Rifkin RM, Gabrail NY, Charu V, Clowney B et al. Phase 3b UPFRONT study: safety and efficacy of weekly bortezomib maintenance therapy after bortezomib-based induction regimens in elderly, newly diagnosed multiple myeloma patients. Blood 2010; 116: (abstract 619).

83 Ludwig H, Durie BG, McCarthy P, Palumbo A, San Miguel J, Barlogie B et al. IMWG consensus on maintenance therapy in multiple myeloma. Blood 2012; 119: 3003-3015.

84 Nair B, van Rhee F, Shaughnessy Jr. JD, Anaissie E, Szymonifka J, Hoering A et al. Superior results of Total Therapy 3 (2003-33) in gene expression profiling-defined low-risk multiple myeloma confirmed in subsequent trial 200666 with VRD maintenance. Blood 2010; 115: 4168-4173.

85 Ludwig H, Adam Z, Tothova E, Hajek R, Labar B, Egyed M et al. Thalidomide maintenance treatment increases progression-free but not overall survival in elderly patients with myeloma. Haematologica 2010; 95: 1548-1554.

86 Attal M, Harousseau JL, Leyvraz S, Doyen C, Hulin C, Benboubker L et al. Maintenance therapy with thalidomide improves survival in patients with multiple myeloma. Blood 2006; 108: 3289-3294.

87 Kaufman JL, Nooka AK, Muppidi S, Heffner LT, Gleason C, Boise L et al. Survival outcomes of early autologous stem cell transplant (ASCT) followed by lenalidomide, bortezomib, and dexamethasone (RVD) maintenance in patients with high-risk multiple myeloma (MM). J Clin Oncol 2012; 30: (abstract 8100).

88 Lonial S, Kaufman JL. The era of combination therapy in myeloma. J Clin Oncol 2012; 30: 2434-2436.

89 Mitsiades N, Mitsiades CS, Poulaki V, Chauhan D, Richardson PG, Hideshima T et al. Apoptotic signaling induced by immunomodulatory thalidomide analogs in human multiple myeloma cells: therapeutic implications. Blood 2002; 99: 4525-4530.

90 Hideshima T, Chauhan D, Shima Y, Raje N, Davies FE, Tai YT et al. Thalidomide and its analogs overcome drug resistance of human multiple myeloma cells to conventional therapy. Blood 2000; 96: 2943-2950.

91 Chauhan D, Tian Z, Zhou B, Kuhn D, Orlowski R, Raje N et al. In vitro and in vivo selective antitumor activity of a novel orally bioavailable proteasome inhibitor MLN9708 against multiple myeloma cells. Clin Cancer Res 2011; 17: 5311-5321.

92 Chauhan D, Singh AV, Ciccarelli B, Richardson PG, Palladino MA, Anderson KC. Combination of novel proteasome inhibitor NPI-0052 and lenalidomide trigger in vitro and in vivo synergistic cytotoxicity in multiple myeloma. Blood 2010; 115: 834-845.

93 Mitsiades N, Mitsiades CS, Richardson PG, Poulaki V, Tai YT, Chauhan D et al. The proteasome inhibitor PS-341 potentiates sensitivity of multiple myeloma cells to conventional chemotherapeutic agents: therapeutic applications. Blood 2003; 101: 2377-2380.

$94 \mathrm{Ma} \mathrm{MH}$, Yang HH, Parker K, Manyak S, Friedman JM, Altamirano C et al. The proteasome inhibitor PS-341 markedly enhances sensitivity of multiple myeloma tumor cells to chemotherapeutic agents. Clin Cancer Res 2003; 9: 1136-1144.

95 Hideshima T, Richardson P, Chauhan D, Palombella VJ, Elliott PJ, Adams J et al. The proteasome inhibitor PS-341 inhibits growth, induces apoptosis, and overcomes drug resistance in human multiple myeloma cells. Cancer Res 2001; 61: $3071-3076$

96 Dimopoulos MA, Kastritis E, Christoulas D, Migkou M, Gavriatopoulou M, Gkotzamanidou $M$ et al. Treatment of patients with relapsed/refractory multiple myeloma with lenalidomide and dexamethasone with or without bortezomib: prospective evaluation of the impact of cytogenetic abnormalities and of previous therapies. Leukemia 2010; 24: 1769-1778.

97 Richardson PG, Jagannath S, Jakubowiak AJ, Lonial S, Raje N, Alsina M et al. Phase II trial of lenalidomide, bortezomib, and dexamethasone in patients (pts) with relapsed and relapsed/refractory multiple myeloma (MM): updated efficacy and safety data after $>2$ years of follow-up. ASH Annual Meeting Abstracts 2010; 116: 3049.

98 Niesvizky R, Martin 3rd TG, Bensinger WI, Alsina M, Siegel DS, Kunkel LA et al. Phase lb dose-escalation study (PX-171-006) of carfilzomib, lenalidomide, and low-dose dexamethasone in relapsed or progressive multiple myeloma. Clin Cancer Res 2013; 19: 2248-2256.

99 Richardson PGG, Berdeja JG, Niesvizky R, Lonial S, Roy V, Hari P et al. Oral weekly MLN9708, an investigational proteasome inhibitor, in combination with lenalidomide and dexamethasone in patients (pts) with previously untreated multiple myeloma (MM): A phase I/II study. J Clin Oncol 2012; 30: (abstract 8033).

100 Kumar S, Flinn I, Richardson PG, Hari P, Callander N, Noga SJ et al. Randomized, multicenter, phase 2 study (EVOLUTION) of combinations of bortezomib, dexamethasone, cyclophosphamide, and lenalidomide in previously untreated multiple myeloma. Blood 2012; 119: 4375-4382.

101 Paiva B, Almeida J, Perez-Andres M, Mateo G, Lopez A, Rasillo A et al. Utility of flow cytometry immunophenotyping in multiple myeloma and other clonal plasma cell-related disorders. Cytometry B Clin Cytom 2010; 78: 239-252.

102 Paiva B, Martinez-Lopez J, Vidriales MB, Mateos MV, Montalban MA Fernandez-Redondo E et al. Comparison of immunofixation, serum free light chain, and immunophenotyping for response evaluation and prognostication in multiple myeloma. J Clin Oncol 2011; 29: 1627-1633.

103 Paiva B, Vidriales MB, Cervero J, Mateo G, Perez JJ, Montalban MA et al. Multiparameter flow cytometric remission is the most relevant prognostic factor for multiple myeloma patients who undergo autologous stem cell transplantation. Blood 2008; 112: 4017-4023.

104 Putkonen M, Kairisto V, Juvonen V, Pelliniemi T, Rauhala A, Itala-Remes M et al. Depth of response assessed by quantitative ASO-PCR predicts the outcome 
after stem cell transplantation in multiple myeloma. Eur J Haematol 2010; 85: 416-423.

105 Martinez-Sanchez P, Montejano L, Sarasquete ME, Garcia-Sanz R Fernandez-Redondo E, Ayala R et al. Evaluation of minimal residual disease in multiple myeloma patients by fluorescent-polymerase chain reaction: the prognostic impact of achieving molecular response. Br J Haematol 2008; 142: 766-774.

106 Corradini P, Voena C, Tarella C, Astolfi M, Ladetto M, Palumbo A et al. Molecular and clinical remissions in multiple myeloma: role of autologous and allogeneic transplantation of hematopoietic cells. J Clin Oncol 1999; 17: 208-215.

107 Rajkumar SV, Harousseau JL, Durie B, Anderson KC, Dimopoulos M, Kyle R et al. Consensus recommendations for the uniform reporting of clinical trials: report of the International Myeloma Workshop Consensus Panel 1. Blood 2011; 117: 4691-4695.

108 Terragna C, Zamagni E, Petrucci MT, Durante S, Patriarca F, Narni F et al. Molecular remission after bortezomib-thalidomide-dexamethasone compared with thalidomide-dexamethasone as consolidation therapy following double autologous transplantation for multiple myeloma: results of a qualitative and quantitative analysis. Blood 2010; 116: (abstract 861).

109 Bensinger W. Stem-cell transplantation for multiple myeloma in the era of novel drugs. J Clin Oncol 2008; 26: 480-492.

110 Lacy MQ, Hayman SR, Gertz MA, Dispenzieri A, Buadi F, Kumar S et al. Pomalidomide (CC4047) plus low-dose dexamethasone as therapy for relapsed multiple myeloma. J Clin Oncol 2009; 27: 5008-5014.

111 Vij R, Wang M, Kaufman JL, Lonial S, Jakubowiak AJ, Stewart AK et al. An open-label, single-arm, phase 2 (PX-171-004) study of single-agent carfilzomib in bortezomib-naïve patients with relapsed and/or refractory multiple myeloma. Blood 2012; 119: 5661-5670.

112 Wolf JL, Siegel D, Goldschmidt H, Hazell K, Bourquelot PM, Bengoudifa BR et al. Phase II trial of the pan-deacetylase inhibitor panobinostat as a single agent in advanced relapsed/refractory multiple myeloma. Leuk Lymphoma 2012; 53 1820-1823.

113 Lonial S, Vij R, Harousseau JL, Facon T, Moreau P, Mazumder A et al. Elotuzumab in combination with lenalidomide and low-dose dexamethasone in relapsed or refractory multiple myeloma. J Clin Oncol 2012; 30: 1953-1959.

114 Dytfeld D, Griffith KA, Friedman J, Lebovic D, Harvey C, Kaminski MS et al. Superior overall survival of patients with myeloma achieving very good partial response or better to initial treatment with bortezomib, pegylated liposomal doxorubicin, and dexamethasone, predicted after two cycles by a free light chain- and M-protein-based model: extended follow-up of a phase II trial. Leuk Lymphoma 2011; 52: 1271-1280.

115 Gertz MA, Kumar S, Lacy MQ, Dispenzieri A, Dingli D, Hayman SR et al. Stem cell transplantation in multiple myeloma: impact of response failure with thalidomide or lenalidomide induction. Blood 2010; 115: 2348-2353.

116 Kapoor P, Kumar S, Dispenzieri A, Lacy M, Dingli D, Hayman SR et al. Survival outcomes of patients with multiple myeloma (MM) achieving stringent complete response (sCR) following upfront autologous stem cell transplantation (SCT). J Clin Oncol 2011; 29: (abstract 8069).

117 Alegre A, Diaz-Mediavilla J, San-Miguel J, Martinez R, Garcia Larana J, Sureda A et al. Autologous peripheral blood stem cell transplantation for multiple myeloma: a report of 259 cases from the Spanish Registry. Spanish Registry for Transplant in MM (Grupo Espanol de Trasplante Hematopoyetico-GETH) and PETHEMA. Bone Marrow Transplant 1998; 21: 133-140.

This work is licensed under a Creative Commons AttributionNonCommercial-NoDerivs 3.0 Unported License. To view a copy of this license, visit http://creativecommons.org/licenses/by-nc-nd/3.0/ 